\title{
Design of Low Voltage Up-conversion Mixer for WSN Applications*
}

\author{
Lei $\mathrm{Wu}$, Zhiqun $\mathrm{Li}^{+}$ \\ Institute of RF- \& OE-ICs, Southeast University, Nanjing, 210096; \\ School of Integrated Circuits, Southeast University, Nanjing, 210096; \\ Key Laboratory of Jiangsu Province Sensor Network Technology, Wuxi, 214135 \\ ${ }^{+}$Corresponding author: zhiqunli@seu.edu.cn
}

\begin{abstract}
A high linearity up-conversion mixer operating at $700 \sim 900 \mathrm{MHz}$ with $1 \mathrm{~V}$ supply using $0.18 \mu \mathrm{m}$ CMOS technology is proposed in this paper. This up-conversion mixer is composed with two double-balanced mixers and is based on the Gilbert mixer. The quadrature differential signals are put to the mixer, and the output of the mixer will be added in the form of current, that achieves the quadrature modulation. The up-conversion mixer takes advantage of current-reuse bleeding, which can improve the conversion gain and reduce the noise figure. In addition, the positive feedback structure is used in this up-conversion mixer so that the conversion gain will be improved further. From the simulation result, the mixer demonstrates $3.39 \mathrm{~dB}$ conversion gain, $0.097 \mathrm{dBm}$ input $1 \mathrm{~dB}$ compression, $9.57 \mathrm{dBm}$ IIP3 and $22.28 \mathrm{~dB}$ noise figure with $1.36 \mathrm{~mW}$ power dissipation from a $1 \mathrm{~V}$ supply.
\end{abstract}

Key words up-conversion mixer; low voltage; current-reuse bleeding; positive feedback

\section{Introduction}

Wireless sensor network is composed of a large number of sensor nodes through wireless communication technology. It is a new information acquisition platform, with the characteristics of low power consumption, low cost, distributed and self-organization. It can real time monitoring and gathering the monitoring information of the object in a particular area. The RF transceiver works as a core

\footnotetext{
* Project supported by the National High Technology Research and Development Program (No. 2007AA01Z2A7) and the Special Fund of Jiangsu Province for the Transformation of Scientific and Technological Achievements (No. BA2010073).
} 
device for the wireless sensor network node, its performance is very important for the communication quality of whole network and the battery endurance ability. The mixer is a core block in RF and analog baseband interface, its circuit design is very important which directly determines the whole performance of the RF transceiver.

The up-conversion mixer is the core module of the transmitter, which the main role is to achieve frequency conversion, converting the baseband signal frequency to the RF output frequency. The performance of the mixer is influenced each other, which means an improvement of one performance will lead to a deterioration of another performance. As the wireless mobile terminal miniaturization, light weight, low voltage and low power applications have become more and more important. As the RF product cost is needed lower, how to use CMOS technology with the advantages of low cost and high degree of integration applied in WSN node becomes the focus of research and design of radio frequency integrated circuits.

Direct up-conversion mixer transmitter has the advantages of simple structure, high integration and low cost, which satisfy the requirements of WSN nodes system. So the direct up-conversion mixer method is used in this system design. The direct up-conversion mixer transmitter structure is shown in Fig.1.

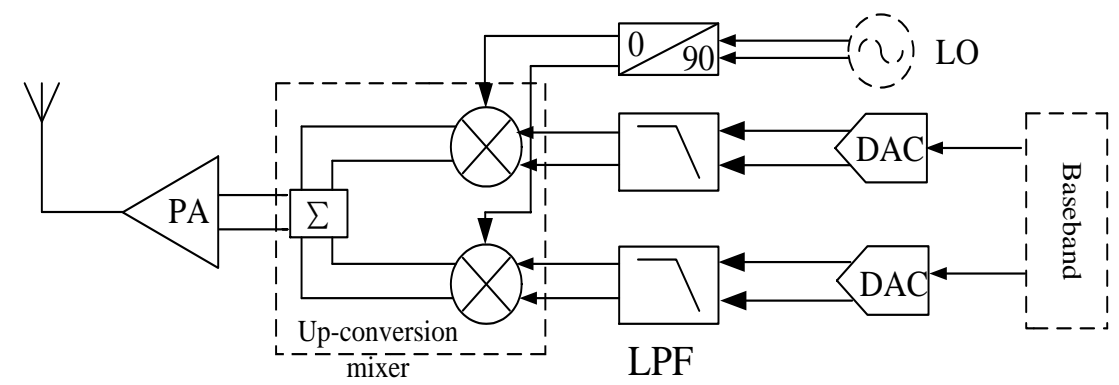

Fig.1 Block diagram of the transmitter

\section{The Traditional Gilbert Mixer}

The traditional Gilbert mixer structure [1-3] is shown in Fig.2. 


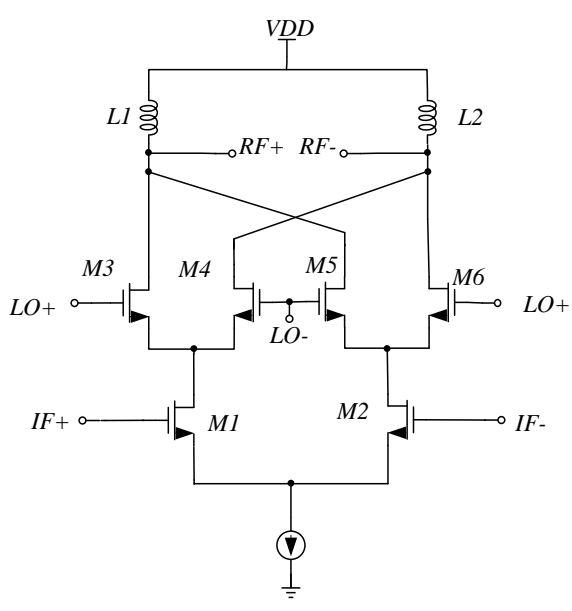

Fig.2 Traditional Gilbert mixer structure

It is composed of the input transconductance stage, the switching stage and the output load. Assume that the input transconductance transistors $\mathrm{M}_{1}$ and $\mathrm{M}_{2}$ work in saturation region and the switching pairs $\mathrm{M}_{3} \sim \mathrm{M}_{6}$ work as switches ideally, current flowing through M1 and M2 respectively is [2]

$$
\begin{aligned}
& i_{\mathrm{M} 1}=I_{\mathrm{IF}}+\frac{1}{2} g_{\mathrm{m} 1} V_{\mathrm{IF}} \cos \omega_{\mathrm{IF}} t \\
& i_{\mathrm{M} 2}=I_{\mathrm{IF}}-\frac{1}{2} g_{\mathrm{m} 2} V_{\mathrm{IF}} \cos \omega_{\mathrm{IF}} t
\end{aligned}
$$

Where $v_{\mathrm{IF}}(t)=V_{\mathrm{IF}} \cos \omega_{\mathrm{IF}} t$.

The RF output current is [2]

$$
i_{\mathrm{O}, \mathrm{RF}} \equiv i_{\mathrm{RF}+}-i_{\mathrm{RF}-}=\left(i_{\mathrm{M} 3}-i_{\mathrm{M} 4}\right)+\left(i_{\mathrm{M} 5}-i_{\mathrm{M} 6}\right)=\left(i_{\mathrm{M} 1}-i_{\mathrm{M} 2}\right) S\left(\omega_{\mathrm{LO}} t\right)
$$

Where $S\left(\omega_{\mathrm{LO}} t\right)$ is the Fourier expansion of $v_{\mathrm{LO}}(t)$.

So the base of the RF output voltage can be written as [2]

$$
V_{\mathrm{O}, \mathrm{RF}}=\frac{2}{\pi} g_{\mathrm{m}, 2} V_{\mathrm{IF}} \omega_{\mathrm{RF}} L \cos \left(\omega_{\mathrm{RF}} t+90^{\circ}\right)
$$

Therefor the conversion gain of Gilbert mixer can be written as [2]

$$
C G=\frac{2}{\pi} g_{\mathrm{m} 1,2} \omega_{\mathrm{RF}} L
$$

\section{Proposed Mixer}

\subsection{Proposed mixer structure}

The proposed mixer structure, which is based on the Gilbert mixer unit, is shown in Fig.3. 


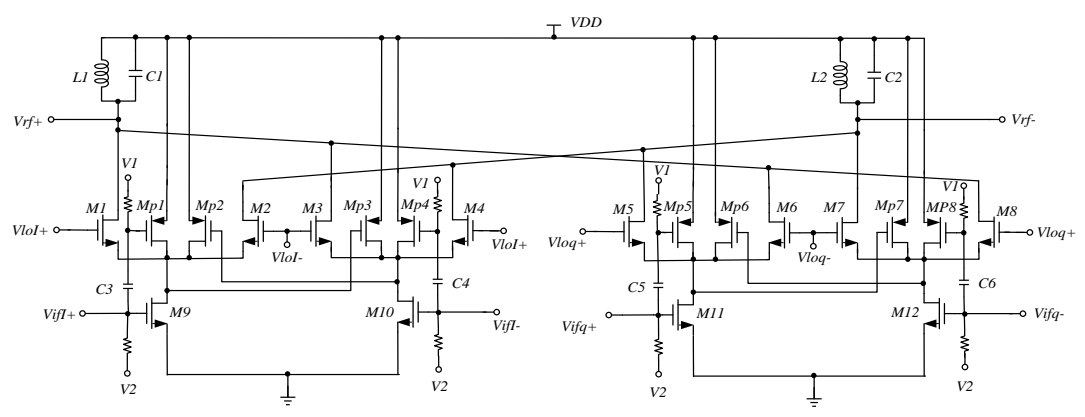

Fig.3 Proposed mixer structure

The up-conversion is composed of two double balanced mixers, their inputs are quadrature differential signals, and their outputs added in the current domain, so that it can realize quadrature modulation. The final output only has the added signal; the minus signal can be suppressed effectively. Ideally, image signal can be completely suppressed by this structure. So the I/Q matching is very important for the quadrature up-conversion mixer.

The current-reuse bleeding [4-5] structure is used in this proposed mixer structure, which can increase the conversion gain and decrease the noise figure. In addition, the positive feedback [6] structure is used in this up-conversion mixer which is between the two switching transistors, so the conversion gain will be improved further.

The output frequency of the up-conversion mixer is $700 \sim 900 \mathrm{MHz}$, so the LC tank can be used in the mixer. The LC tank won't produce dc voltage drop, it can relax voltage headroom issues and increase the dynamic range of the output voltage. What's more, the LC tank can provide higher impedance, so that the mixer can get a higher conversion gain. In addition, the LC tank has the characteristic of filtering, which can reject the other harmonic component except useful RF frequency.

\subsection{Analysis of the circuit}

\subsubsection{Current-reuse bleeding structure}

The linearity of the input transistor is positive to the square root of the tail current of Gilbert mixer [7]. So the linearity of the mixer can be improved by increasing the current of the input transconductor. But infect that the increasing of the current of the input transconductor can lead to the increasing of the current of the switching stage and the load resistance. The noise of the switching stage can be 
increased by the increasing of the current of the switching stage. So the current-bleeding method is used to solve the contradiction of the linearity and the noise. Its mean idea is to only increase the input current, without increasing the switching stage and a load current by the bleeding of the current. The current-bleeding structure is shown in Fig.4 [5].

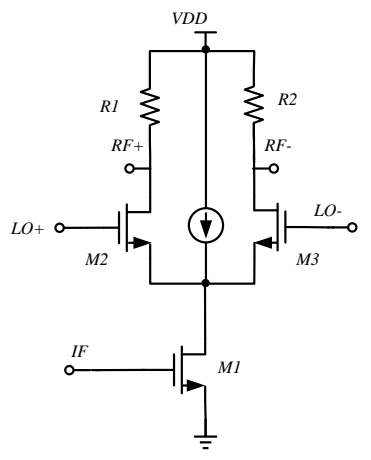

(a)

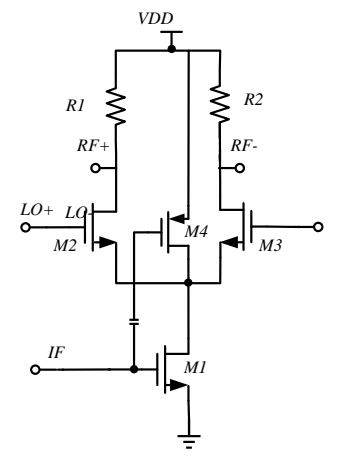

(b)

Fig.4 (a) traditional current-bleeding structure

(b) current-reuse bleeding structure

The current-reuse bleeding structure is based on the traditional current-bleeding structure, just as shown in Fig.4 (b). The current-reuse bleeding structure is composed with M1 and M4. The PMOS added between the switching transistors works as the injected current source as well as a part of the driving amplifier. At the same condition of voltage gain, its power consumption can be fallen by half in theory compared with the traditional Gilbert mixer. The conversion gain is improved by increasing the current of transconductor stage. Because the PMOS improves the transconductance by reusing the current of NMOS without increasing extra power dissipation, which makes the general transconductance is equal to the sum of two tube transconductance. This new structure has the advantage of high conversion gain and low noise figure.

\subsubsection{Positive feedback structure}

A positive feedback structure can be formed by connecting the gate of one MOSFET to the drain of another MOSFET respectively, just as shown in Fig.5. 


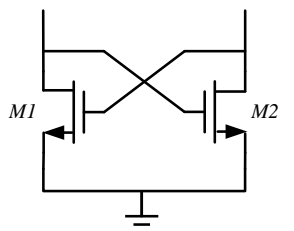

(a)

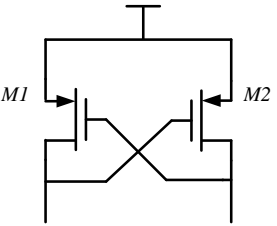

(b)

Fig.5 (a) NMOS positive feedback

(b) PMOS positive feedback

The differential signals are put into the gate of the M1 and M2 respectively, the drain of the M1 and M2 will output the signals that the phase is opposite to the input signal. Through this cross connection, the output of the transistor will get the same phase signal that can enhance the signal of output. Hence, this structure can generate greater output signal at the same input signal, and then bring to the input, that forms the positive feedback. From another point of view, this positive feedback can generate a negative resistance $-2 / \mathrm{gm}$, which will be connected in parallel with the load resistance. So the total load will be increased, and the conversion gain will be improved.

On the other hand, the positive feedback structure is put between the two switching stages in this design. It will not increase the total current which makes the low power come true.

\section{Post Simulation Results}

The mixer is designed in $0.18 \mu \mathrm{m}$ RF CMOS process. Its layout is shown in Fig.6, which total area is $1.595 \times 1.283 \mathrm{~mm}^{2}$.

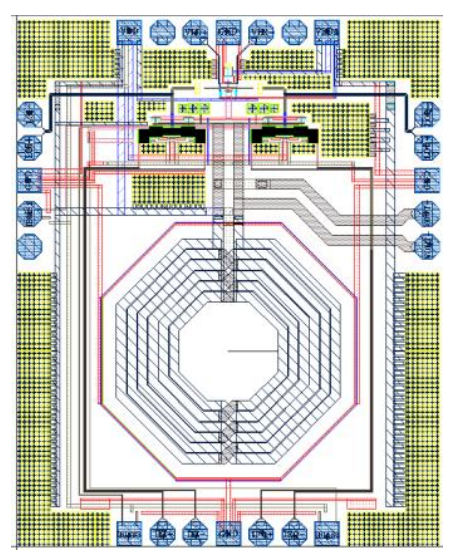

Fig.6 layout of the mixer 
The mixer is simulated with Cadence specter simulator. Its input base band signal frequency is $10 \mathrm{MHz}$ and amplitude is $-30 \mathrm{dBm}$. The LO signal frequency is $790 \mathrm{MHz}$ and amplitude is $0 \mathrm{dBm}$. The process corner is TT and the temperature is $27^{\circ} \mathrm{C}$.

The simulation results are shown from Fig. 7 (a) to (e). The mixer achieves $3.394 \mathrm{~dB}$ of conversion gain, $0.0969 \mathrm{dBm}$ of input referred $1 \mathrm{~dB}$ compression point, $9.57 \mathrm{dBm}$ of IIP3 and $22.28 \mathrm{~dB}$ of noise figure.
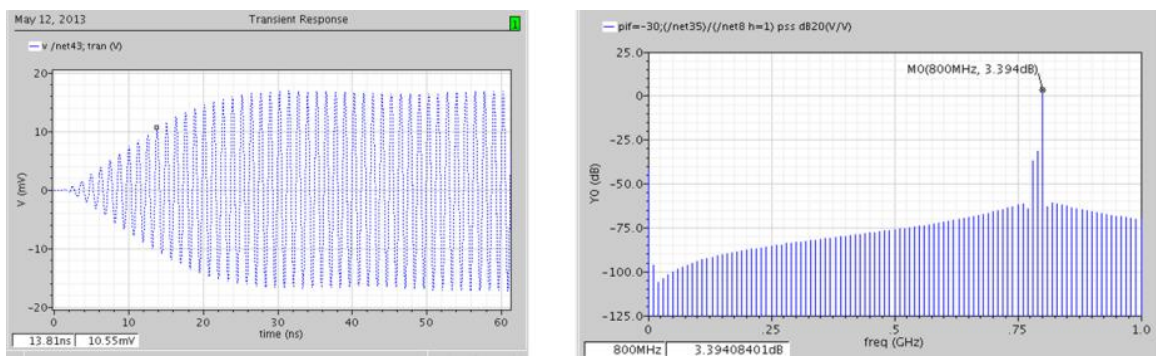

Fig.7 (a) frequency domain diagram of RF output

Fig.7 (b) conversion gain

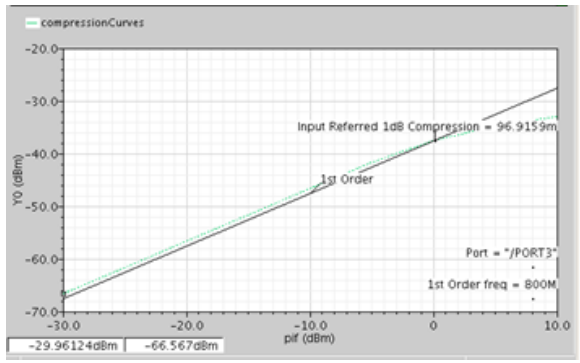

Fig.7 (c) input referred $1 \mathrm{~dB}$ compression point

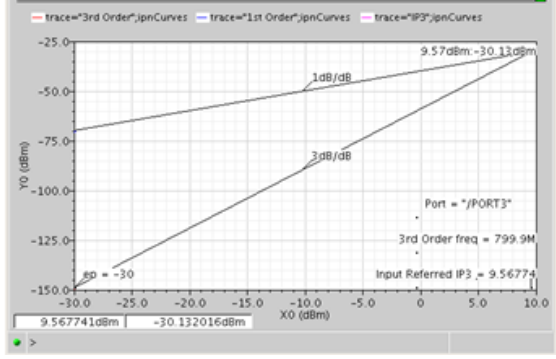

Fig.7 (d) IIP3

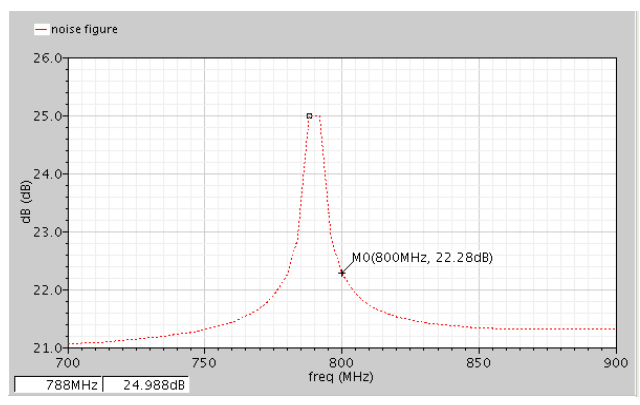

Fig.7 (e) noise figure 


\section{Conclusions}

This paper introduces a kind of low voltage quadrature up-conversion mixer which is applied in WSN node transmitter system. The quadrature modulation can reject image signal, the current-reuse bleeding structure and positive feedback structure can improve the conversion gain and reduce the noise figure.

\section{References}

[1] Li Zhiqun, Wang Zhigong, Radio Frequency Integrated Circuits and System, 2008

[2] Chi Baoyong, Yu Zhiping, Shi Bingxue. The Design and Analysis of CMOS Radio-Frequency Integrated Circuits [M]. 2006

[3] Thomas $\mathrm{H}$ lee, The Design of CMOS Radio-Frequency Integrated Circuits [M]. Second Edition. Cambridge University Press, 2003

[4] Lee, S.G., Choi, J.-K. Current-reuse bleeding mixer [J]. Electronics Letters, 2000, 36(8): 696-697.

[5] Tu, S.H.-L., Sky Chang-Hung Chen. A 5.26-GHz CMOS Up-Conversion Mixer for IEEE 802.11a WLAN [J]. Circuits and Systems for Communications,2008,820-823

[6] Chang-His Wu, Wen-Hui Huang. A high-linearity up-conversion mixer utilizing negative resistor [J]. International Symposium on Signals Systems and Electronics (ISSSE), 2010, 2: 1-4

[7] Yu C, Yuan J S. Linearity and power optimization of a microwave CMOS Gilbert cell mixer[C]. Electron Devices for Microwave and Optoelectronic Applications 